\title{
Periprosthetic Fracture Resembling Atypical Femoral Fracture After Fixation With Retrograde Intramedullary Nail in Elderly Women: A Report of Two Cases
}

\author{
YOJIRO TAKAHASHI ${ }^{1,2,3}$, SATOSHI HATASHITA $^{1,2}$, YUMETAKA SHINDEN $^{1,2,3}$, \\ MASAYUKI ITO ${ }^{1,2}$, YOICHI KANEUCHI ${ }^{3}$, MICHIYUKI HAKOZAKI $^{3,4}$ and SHINICHI KONNO ${ }^{3}$ \\ ${ }^{1}$ Aizu Traumatology and Reconstructive Surgery Center, Aizu Chuo Hospital, Fukushima, Japan; \\ ${ }^{2}$ Department of Traumatology and Reconstructive Surgery, \\ Fukushima Medical University School of Medicine, Fukushima, Japan; \\ ${ }^{3}$ Department of Orthopaedic Surgery Fukushima Medical University School of Medicine, Fukushima, Japan; \\ ${ }^{4}$ Higashi-Shirakawa Orthopaedic Academy, Fukushima Medical University School of Medicine, Fukushima, Japan
}

\begin{abstract}
Background/Aim: The number of individuals diagnosed with and requiring medical treatment for osteoporosis continues to increase due to global population aging and the high awareness of osteoporosis. Bone-modifying agents (BMAs) including bisphosphonate and denosumab are widely used for osteoporosis, and atypical femoral fracture $(A F F)$ is also gaining attention as a severe potential side effect of long-term BMA treatment. The definition of AFF excludes periprosthetic femoral fracture; here, we describe two cases of a periprosthetic femoral fracture that resembled AFF. Case Report: The fractures occurred at the proximal tip of the retrograde femoral nail after an internal fixation for a distal femoral shaft fracture in elderly Japanese women. Each woman had been treated with bisphosphonate therapy for $>2$ years and had continued the bisphosphonate after undergoing surgery for a distal femoral shaft fracture. Each patient had
\end{abstract}

This article is freely accessible online.

This article is based on the study first reported in the Journal of Japanese Society for Fracture Repair: Tahahashi M, Hatashita S, Ito $\mathrm{M}$ and Shinden $\mathrm{Y}$ : The problem after the treatment of retrograde femoral nail to the aged: 2 cases of the femoral subtrochanteric fracture at the proximal end of retrograde femoral nail. J Jpn Soc Fracture Repair 42(2): 1303-1308, 2020 (in Japanese).

Correspondence to: Dr. Michiyuki Hakozaki, Department of Orthopaedic Surgery, Fukushima Medical University School of Medicine, 1 Hikarigaoka, Fukushima-shi, Fukushima 960-1295, Japan. Tel: +81 245471276, Fax: +81 245485505, email: paco@fmu.ac.jp

Key Words: Atypical femoral fracture, periprosthetic fracture, bonemodifying agent, retrograde intramedullary nail, elderly patient. noticed thigh pain before falling down, and plain radiographs showed a short oblique or transverse fracture with medial spike and localized periosteal reaction of the lateral cortex in each case. The fractures were re-fixed with an antegrade intramedullary nail, and bone union was achieved at $>1$ year after the second operation. Although these two cases were classified as periprosthetic fractures, they fulfilled the characteristics of AFF. Conclusion: Physicians should conduct a thorough interview of patients with a history of BMA treatment in order to correctly diagnose periprosthetic fractures that resemble AFFs, and they should be aware that symptomatic fractures can be prevented by prophylactic fixation. The discontinuance of BMA therapy and the introduction of another drug such as teriparatide may lead to faster healing of surgically treated AFFs.

The number of individuals diagnosed with and receiving medical treatment for osteoporosis has markedly and rapidly increased as lifespans continue to expand (1). Bonemodifying agents (BMAs) including bisphosphonate and denosumab are the representative drugs for treating osteoporosis and are now used globally. One of the potential adverse effects of long-term BMA treatment is atypical femoral fracture (AFF), which is also gathering attention. Herein, we describe two cases of a femoral subtrochanteric fracture resembling an AFF that occurred at the proximal tip of the retrograde intramedullary nail (IMN) after the internal fixation for a femoral distal shaft fracture in elderly patients.

\section{Case Presentation}

Case 1. An 81-year-old Japanese woman presented with right thigh pain after falling to the floor. She had taken 10 mg per day of prednisolone for rheumatoid arthritis and 


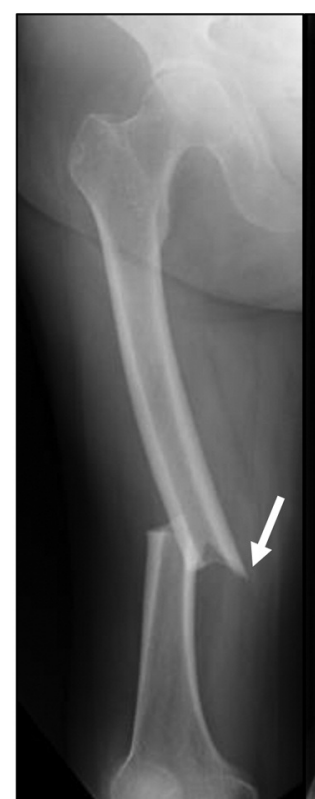

A

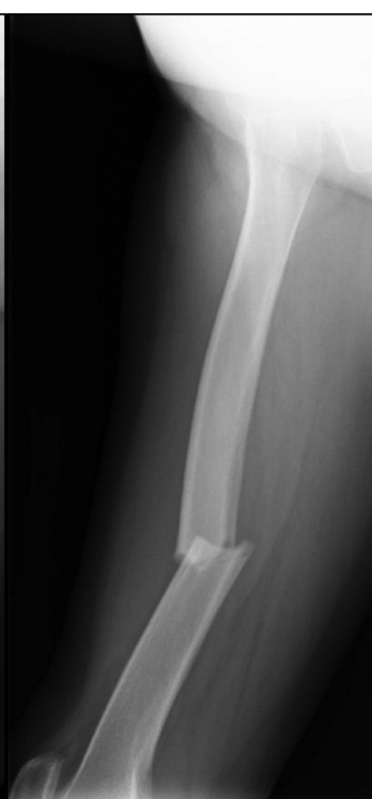

B

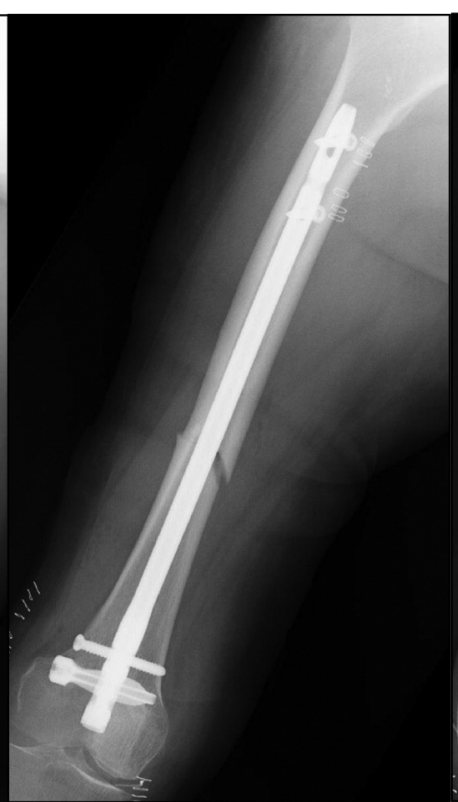

C

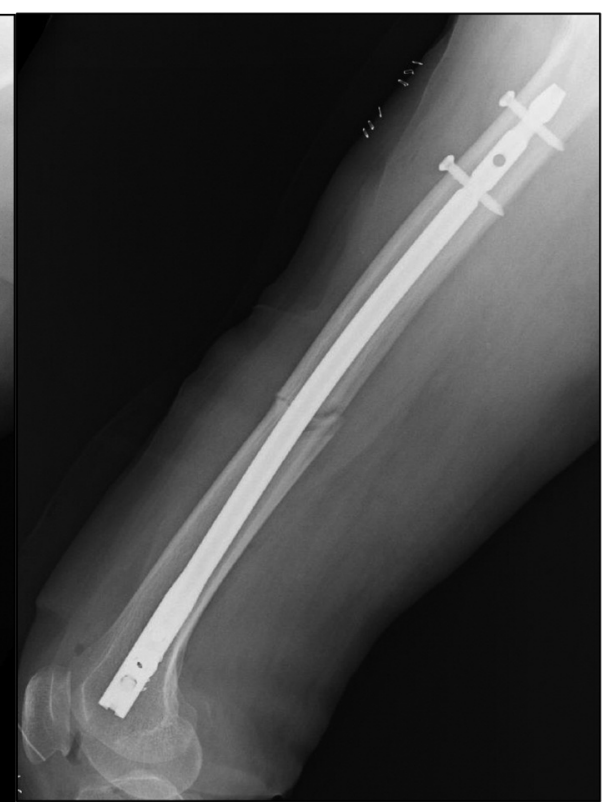

D

Figure 1. Case 1. Plain radiographs of the right femur at the time of the first injury (A, anteroposterior view; $B$, lateral view) showing the transverse distal shaft fracture with the major radiographic feature of $A F F$, i.e. a medial spike (arrow). This fracture was treated surgically by open reduction and internal fixation using a retrograde intramedullary nail (C, anteroposterior view; $D$, lateral view).

bisphosphonate therapy for 8 years to prevent glucocorticoidinduced osteoporosis. The bone mineral density (BMD) values at the lumbar spine and femoral neck were $59 \%$ and $66 \%$ of the young adult mean (YAM), respectively. Plain radiographs showed a right femoral shaft fracture, noncomminuted and of transverse type (Figure 1A, B). The patient was diagnosed with an AFF based on the 2010 criteria issued by the American Society for Bone and Mineral Research (ASBMR) (Table I) (2). She underwent open reduction and internal fixation using a retrograde IMN (Figure 1C, D). She stopped taking the bisphosphonate and received teriparatide treatment for the delayed union, and the fracture finally achieved bone union. At approximately 20 months after the operation, the patient fell to the floor; she reported having felt right thigh pain just before falling.

Plain radiographs showed a right femoral subtrochanteric fracture at the level of the most proximal transverse screw of the retrograde IMN. It was a non-comminuted and short oblique fracture with a medial spike and a localized periosteal reaction (flaring) of the lateral cortex (Figure 2A, B). Although this fracture resembled an AFF, it was classified as a periprosthetic fracture due to the AFF exclusion criteria. The retrograde IMN was removed, and the fracture was re-fixed with an antegrade IMN. After the second operation, the oral bisphosphonate re-started by the patient's physician after the first operation was stopped again, and teriparatide treatment was re-started. Low-
Table I. The criteria of atypical femoral fracture (AFF).

Major and minor features ${ }^{\mathrm{a}}$

Major features: ${ }^{b}$

- Located anywhere along the femur from distal to the lesser trochanter to proximal to the supracondylar flare

- Associated with no trauma or minimal trauma, as in a fall from a standing height or less

- Transverse or short oblique configuration

- Noncomminuted

- Complete fractures extend through both cortices and may be associated with a medial spike; incomplete fractures involve only the lateral cortex

Minor features:

- Localized periosteal reaction of the lateral cortex ${ }^{\mathrm{c}}$

- Generalized increase in cortical thickness of the diaphysis

- Prodromal symptoms such as dull or aching pain in the groin or thigh

- Bilateral fractures and symptoms

- Delayed healing

- Comorbid conditions (e.g., vitamin D deficiency, rheumatoid arthritis, hypophosphatasia)

- Use of pharmaceutical agents (e.g., bisphosphonates, glucocorticoids, proton pump inhibitors)

aFractures of the femoral neck, intertrochanteric fractures with spiral subtrochanteric extension, pathologic fractures associated with primary or metastatic bone tumors, and periprosthetic fractures are excluded. ${ }^{b}$ All major features are required to satisfy the case definition of AFF. None of the minor features are required but sometimes they have been

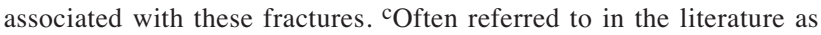
beaking or flaring. Source: 2010 ASBMR (2). 


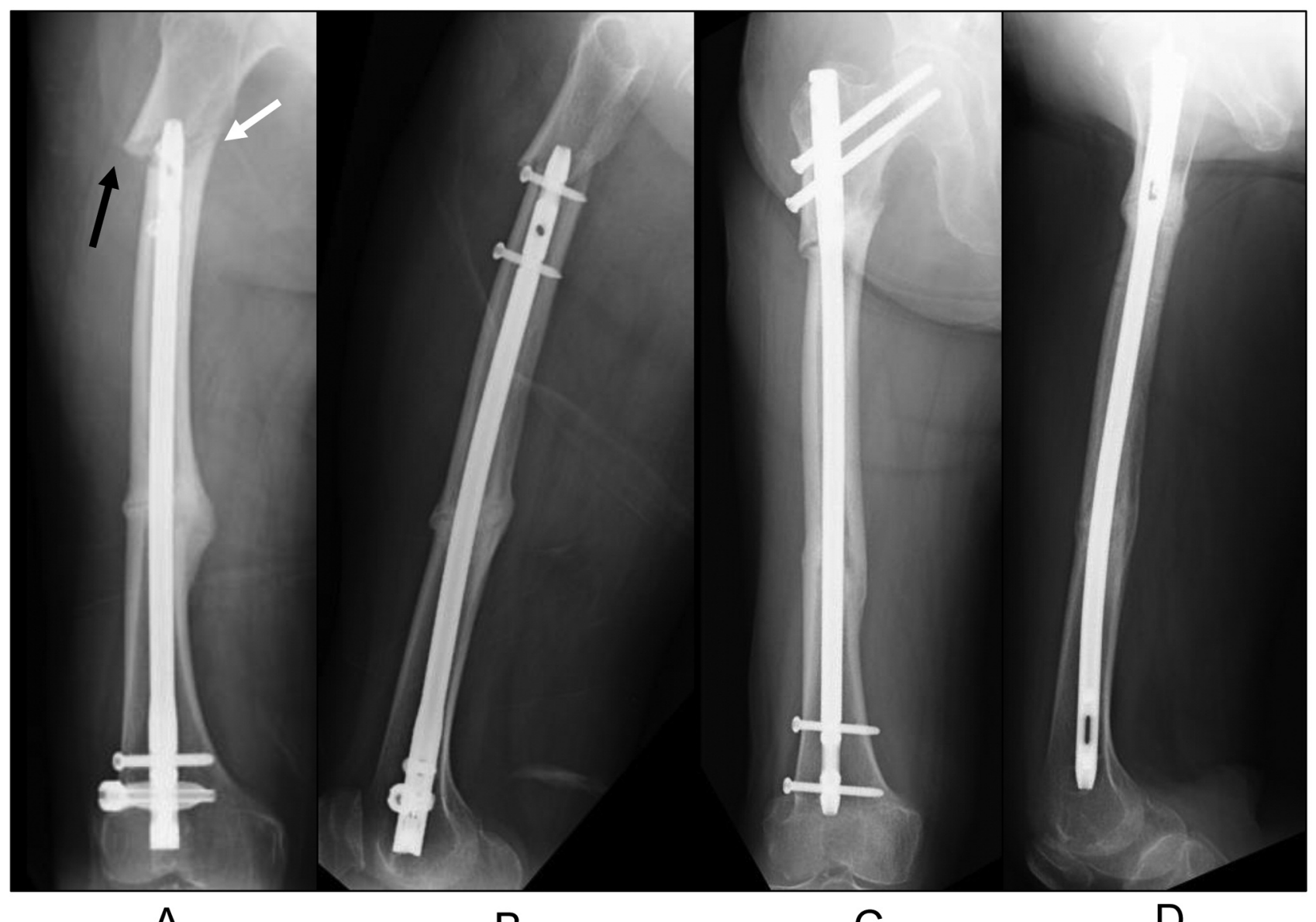

A

B

C

Figure 2. Case 1. Plain radiographs of the right femur demonstrating the subtrochanteric fracture at the level of most proximal transverse screw of the retrograde intramedullary nail ( $A$, anteroposterior view; $B$, lateral view). This was a non-comminuted and short oblique fracture with a medial spike (white arrow) and a localized periosteal reaction (flaring) of the lateral cortex (black arrow). Plain radiographs 2 years after the second operation showing the fracture healing achieved by open reduction and internal fixation using an antegrade intramedullary nail (C, anteroposterior view; $D$, lateral view).

intensity pulsed ultrasound (LIPUS) therapy was used for the prevention of a delayed union. However, 2 months later, the teriparatide treatment was discontinued because of the development of diarrhea and slight fever.

Thereafter, only the oral administration of active vitamin D was continued for the treatment of osteoporosis. Although bone union was delayed at first, it was finally achieved 2 years after the second operation (Figure 2C, D). The patient was able to walk painlessly with a walker, 6 months after the second operation.

Case 2. A 76-year-old Japanese woman who was diagnosed with osteoporosis and had received bisphosphonate therapy for $>2$ years presented to the prior hospital with right thigh pain after falling backward. The BMD values at the lumbar spine and femoral neck were $82 \%$ and $75 \%$ of the YAM, respectively. Plain radiographs showed a right femoral distal shaft fracture, of spiral type. This fracture did not fulfil the criteria for an AFF (Figure 3A, B). The patient underwent open reduction and internal fixation using a retrograde IMN at the prior hospital (Figure 3C, D). The patient's bisphosphonate therapy was continued after the operation, and the bone union was achieved 5 months later.

Three years after the operation, the patient fell again. She also reported feeling right thigh pain before the second fall. Plain radiographs showed a right femoral subtrochanteric fracture at the level of the most proximal transverse screw of the retrograde IMN, revealing a non-comminuted and transverse fracture with a medial spike and a localized periosteal reaction (flaring) of the lateral cortex (Figure 4A, B). Although this fracture resembled an AFF, it was also classified as a periprosthetic fracture because of the AFF exclusion criteria. The retrograde IMN was removed and refixation with antegrade IMN was performed. 


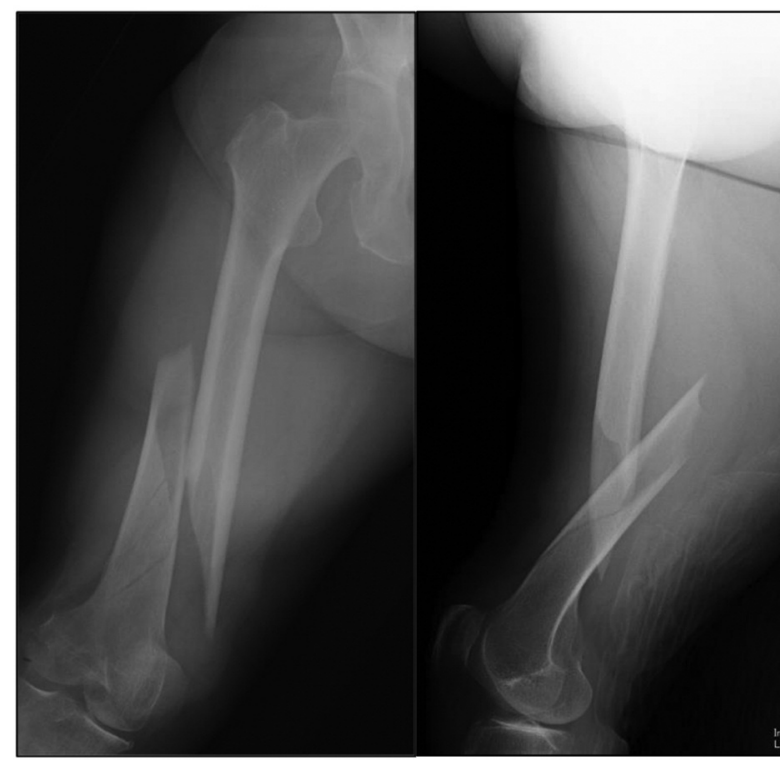

A
B

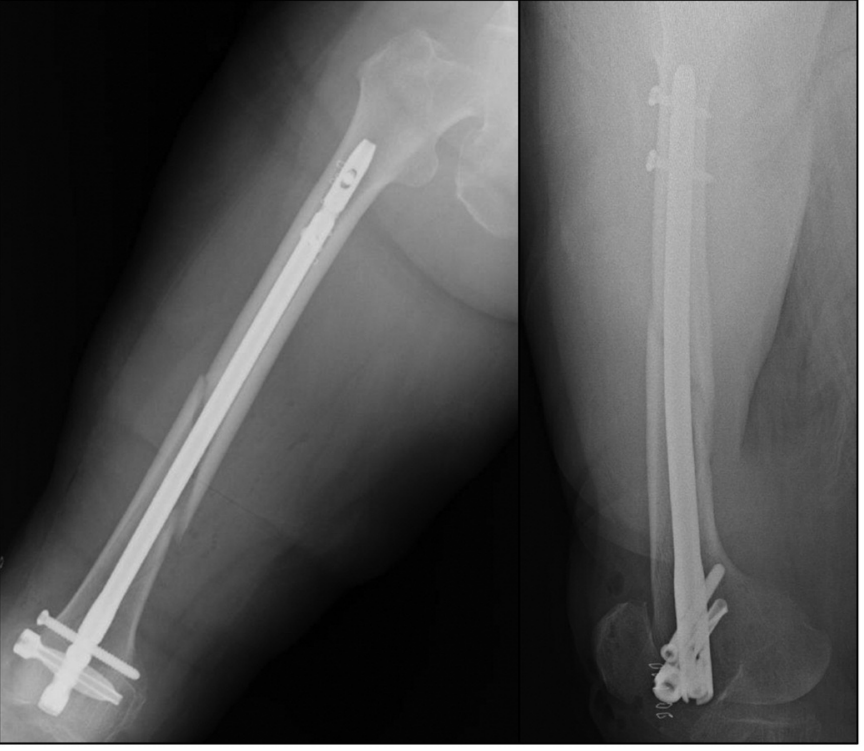

C
$\mathrm{D}$

Figure 3. Case 2. Plain radiographs of the right femur taken at the time of the first injury (A, anteroposterior view; $B$, lateral view) showing the spinal distal shaft fracture. This fracture did not fulfil the criteria of AFF. The fracture was treated surgically by open reduction and internal fixation using a retrograde intermedullary nail ( $C$, anteroposterior view; $D$, lateral view).

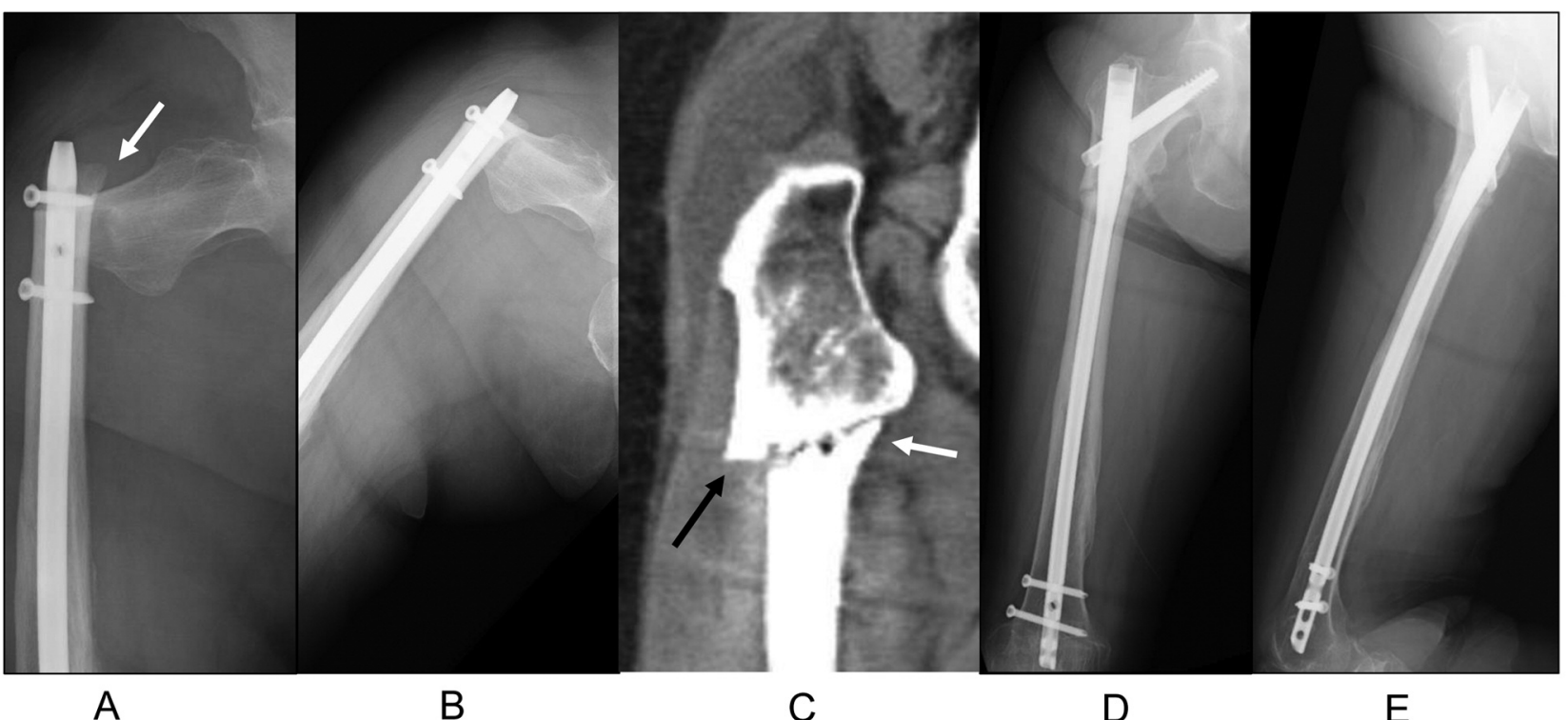

Figure 4. Case 2. Plain radiographs (A, anteroposterior view) and computed tomography image (B, coronal view) of the right femur demonstrating the subtrochanteric fracture at the level of most proximal transverse screw of the retrograde intermedullary nail. This was a non-comminuted and transverse fracture with a medial spike (white arrow) and a localized periosteal reaction (flaring) of the lateral cortex (black arrow). Plain radiographs 1 year after the second operation showing the fracture healing achieved by open reduction and internal fixation using an antegrade intermedullary nail (C, anteroposterior view; $D$, lateral view).

After the second operation, the patient's bisphosphonate therapy was discontinued and both teriparatide and LIPUS therapies were started. Although the bony gap at the lateral cortex remained, bone union was achieved 1 year after the second operation (Figure 4C, D). The patient was able to walk painlessly without a cane or orthosis 3 months after the second operation. 


\section{Discussion}

Due to the significant increase in the number of individuals with osteoporosis, the use of BMAs (including bisphosphonate and denosumab) has also increased, and AFF as a potential side effect of long-term BMA therapy is observed more frequently. An AFF is a femoral subtrochanteric or shaft fracture associated with long-term BMA treatment. The main factor of AFF is thought to be severely suppressed bone turnover (3). In addition, AFF is reported as a type of stress fracture associated with a bowing deformity of the femoral shaft (4). Although a periprosthetic femoral fracture is excluded by the definition of AFF, the number of reports of a periprosthetic femoral fracture harboring the characteristics of AFF is increasing. Niikura et al. (5) reported a case of bisphosphonate-associated AFF that occurred around the femoral component of a total hip arthroplasty. Tanaka et al. (6) reported a similar case, and they suspected that the fracture was influenced by both stress force related to an implant and lateral bowing concentrating on the fracture site as a mechanical factor (in addition to bisphosphonates as a biological factor).

The present two patients were elderly women who had been treated with an oral BMA for osteoporosis for $>5$ years. Their second fractures were diagnosed as periprosthetic fractures resembling AFF. These fractures were influenced by a mechanical factor, i.e. stress force concentrated on the femoral sub-trochanter caused by the retrograde femoral nail, in addition to the biological factor of the BMA. To the best of our knowledge, there is no report of such periprosthetic fractures resembling AFF that occurred around an IMN.

The number of femoral distal shaft fractures including femoral supracondylar fractures in the elderly is expected to increase. Although many studies have reported the efficacy of a retrograde IMN for these fractures (7-10), these can now be securely fixed, due to the improved mechanical stability of antegrade IMN with three or four poly-axial distal transverse screws (11). From the experience of our two cases, we recommend that orthopedic surgeons should take the risk of a periprosthetic femoral subtrochanteric fracture in elderly patients into account in the presurgical planning for an antegrade IMN.

It has been reported that some patients with an AFF may have a prodrome such as thigh pain despite the poor radiographic findings (12). In cases of a periprosthetic fracture resembling AFF, the same problem may be expected as in the present two cases. Physicians can correctly diagnose a periprosthetic fracture resembling an AFF by conducting a thorough interview of the patient about his or her BMA history and surgeons can prevent a symptomatic fracture by performing a prophylactic fixation with an antegrade IMN. After the diagnosis, an early discontinuance of the BMA treatment and the introduction of another drug such as teriparatide may lead to faster healing of surgically treated AFFs $(13,14)$.

\section{Conclusion}

We described two cases of a femoral subtrochanteric fracture around the proximal end of the retrograde femoral nail after internal fixation for a femoral distal shaft fracture in elderly women. Although these were classified as periprosthetic fractures and excluded from AFF by the current diagnostic criteria, they were thought to be fractures resembling an AFF based on the patients' history of long-term bisphosphonate treatment and the characteristics of the fractures. We suggest that femoral distal shaft fractures in elderly individuals with a history of BMA therapy should be treated with an antegrade IMN if possible, to avoid the occurrence of a periprosthetic fracture resembling an AFF.

\section{Conflicts of Interest}

The Authors state that they have no conflicts of interest to declare in regard to this study.

\section{Authors' Contributions}

YT operated on the patient and wrote the manuscript. SH, YS and MI were involved in patient care, manuscript preparation and review. YK, MH and SK were involved in manuscript preparation and review. All Authors read and approved the final manuscript.

\section{References}

1 Orimo H: Japanese 2015 Guidelines for Prevention and Treatment of Osteoporosis. Japan, Life Science Publishing Co., Ltd.,2015 (in Japanese).

2 Shane E, Burr D, Ebeling PR, Abrahamsen B, Adler RA, Brown TD, Cheung AM, Cosman F, Curtis JR, Dell R, Dempster D, Einhorn TA, Genant HK, Geusens P, Klaushofer K, Koval K, Lane JM, McKiernan F, McKinney R, Ng A, Nieves J, O'Keefe R, Papapoulos S, Sen HT, van der Meulen MC, Weinstein RS, Whyte M and American Society for Bone and Mineral Research: Atypical subtrochanteric and diaphyseal femoral fractures: Report of a task force of the American society for bone and mineral research. J Bone Miner Res 25(11): 2267-2294, 2010. PMID: 20842676. DOI: 10.1002/jbmr.253

3 Odvina CV, Zerwekh JE, Rao DS, Maalouf N, Gottschalk FA and Pak CY: Severely suppressed bone turnover: A potential complication of alendronate therapy. J Clin Endocrinol Metab 90(3): 1294-1301, 2005. PMID: 15598694. DOI: 10.1210/ jc.2004-0952

4 Oh Y, Wakabayashi Y, Kurosa Y, Ishizuki M and Okawa A: Stress fracture of the bowed femoral shaft is another cause of atypical femoral fracture in elderly Japanese: A case series. J Orthop Sci 19(4): 579-586, 2014. PMID: 24789301. DOI: 10.1007/s00776-014-0572-9

5 Niikura T, Lee SY, Sakai Y, Kuroda R and Kurosaka M: Rare non-traumatic periprosthetic femoral fracture with features of an atypical femoral fracture: A case report. J Med Case Rep 9: 103, 2015. PMID: 25943378. DOI: 10.1186/s13256-015-0590-z 
6 Tanaka S, Fukui T, Oe K, Matsumoto T, Matsushita T, Hayashi S, Kawamoto T, Kuroda R and Niikura T: A periprosthetic femoral fracture with characteristics of atypical femoral fracture. Case Rep Orthop 2019: 1275369, 2019. PMID: 31396425. DOI: $10.1155 / 2019 / 1275369$

7 Terao M, Hayashi M, Imatani J, Noda T, Shimamura Y, Morito $\mathrm{Y}$ and Nagano H: Comparison - with histories of treatment orthodromic intramedullary nail of the antidromic intramedullary nail for the shaft of femur fracture. J Jpn Soc Fracture Repair 27(2): 606-608, 2005 (in Japanese).

8 Nakamura H, Yoshida K, Yamashita H, Hoshiko H, Yamada K, Watanabe Y, Nagata T, Nago T, Beppu S, Tanaka J and Goto T: Comparison with the antidromic intramedullary nail fixation for the shaft of femur fracture and the orthodromic intramedullary nail fixation. J Jpn Soc Fracture Repair 26(2): 612-615, 2004 (in Japanese).

9 Moed BR and Watson JT: Retrograde intramedullary nailing, without reaming, of fractures of the femoral shaft in multiply injured patients. J Bone Joint Surg Am 77(10): 1520-1527, 1995. PMID: 7593060. DOI: 10.2106/00004623-199510000-00006

10 Ostrum RF, Agarwal A, Lakatos R and Poka A: Prospective comparison of retrograde and antegrade femoral intramedullary nailing. J Orthop Trauma 14(7): 496-501, 2000. PMID: 11083612. DOI: $10.1097 / 00005131-200009000-00006$

11 Ohno M, Nagano H, Kawasaki K, Touno M, Tamura R and Sasaki $\mathrm{K}$ : Histories of treatment and problems in the orthodromic intramedullary nail for thighbone infra-isthmal fracture. J Jpn Soc Fracture Repair 36(3): 682-684, 2014 (in Japanese).
12 Kobayashi K, Kaneuchi Y, Hakozaki M, Otani K and Konno S: Magnetic resonance imaging findings in the hyperacute phase of atypical femoral fracture: A case description. Quant Imaging Med Surg 10(12): 2366-2369, 2020. PMID: 33269232. DOI: 10.21037/qims-20-794

13 van de Laarschot DM, McKenna MJ, Abrahamsen B, Langdahl B, Cohen-Solal M, Guañabens N, Eastell R, Ralston SH and Zillikens MC: Medical management of patients after atypical femur fractures: A systematic review and recommendations from the European calcified tissue society. J Clin Endocrinol Metab 105(5): 1682-1699, 2020. PMID: 31867670. DOI: 10.1210/clinem/dgz295

14 Koh A, Guerado E and Giannoudis PV: Atypical femoral fractures related to bisphosphonate treatment: Issues and controversies related to their surgical management. Bone Joint $\mathrm{J}$ 99-B(3): 295-302, 2017. PMID: 28249967. DOI: 10.1302/0301620X.99B3.BJJ-2016-0276.R2
Received March 8, 2021

Revised March 26, 2021

Accepted March 29, 2021 